\title{
Latest theory developments for top pair production, generators and showering
}

\section{Emanuele $\mathbf{R e}^{*}$}

Rudolf Peierls Centre for Theoretical Physics, University of Oxford, 1 Keble Road, Oxford, UK

Laboratoire d'Annecy-le-Vieux de Physique Theorique (LAPTh),

9 chemin de Bellevue, Annecy-le-Vieux, France ${ }^{\dagger}$

E-mail: emanuele.redlapth.cnrs.fr

I summarize the state of the art of cross-section computations and of available simulation tools for top-quark pair production in hadron collisions. Particular emphasis is put on recent theory developments relevant for LHC phenomenology.

8th International Workshop on Top Quark Physics

14-18 September, 2015

Ischia, Italy

* Speaker.

${ }^{\dagger}$ current address 


\section{Introduction}

Top Physics is a central part of the LHC Physics programme, as shown for instance by the variety of topics and results presented at this conference. The large value of the cross sections to produce top quarks at the LHC allows an experimentally accurate extraction of its properties. On the other hand, the final state arising from its decay products makes top-quark production processes a background for several BSM searches. For these reasons it is extremely important to have predictions for total and differential cross sections that are precise enough to match the experimental accuracy. It is also crucial to model as accurately as possible subleading effects by including higher-order corrections into event generator programs.

In this paper I review the state of the art of computations and simulation tools for top-quark pair production, that is the dominant mechanism for producing top quarks at the LHC. In section 2 I focus on results for total and differential cross sections at parton level, whereas section 3 reviews the current research activity devoted to the improvement of simulation tools.

\section{Recent improvements in the computation of total and differential cross sections}

In this section I will first summarize recent highlights in the computation of the $t \bar{t}$ total and differential cross sections in QCD (sec. 2.1-2.2). In the last part (sec. 2.3) I give the state of the art for fully differential predictions where top quark decay products are included.

\subsection{Fixed order results}

A landmark result for top quark Physics at the LHC was obtained by the authors of ref. [1], that have computed for the first time the exact next-to-next-to-leading order (NNLO) QCD corrections to the total inclusive cross section $\sigma_{t o t}$, using the subtraction scheme presented in ref. [2]. These results show a fairly good convergence of the perturbative series, with good overlap among uncertainty bands when going from one order to the next one. The agreement between theory and data is also remarkably good, as shown in several talks at this conference.

Differential distributions for $t \bar{t}$ production are now also known at NNLO in QCD. They were first published for the forward backward top asymmetry in ref. [3], and, very recently, for other (more exclusive) observables [4]. The importance of the latter results can not be underestimated. NNLO/NLO differential $\mathrm{K}$ factors are generally not flat, as illustrated for instance the plot in fig. 1, where it is shown that NNLO corrections change sizeably the shape of the top quark $p_{T}$ spectrum. ${ }^{1}$ In the tail region several studies by

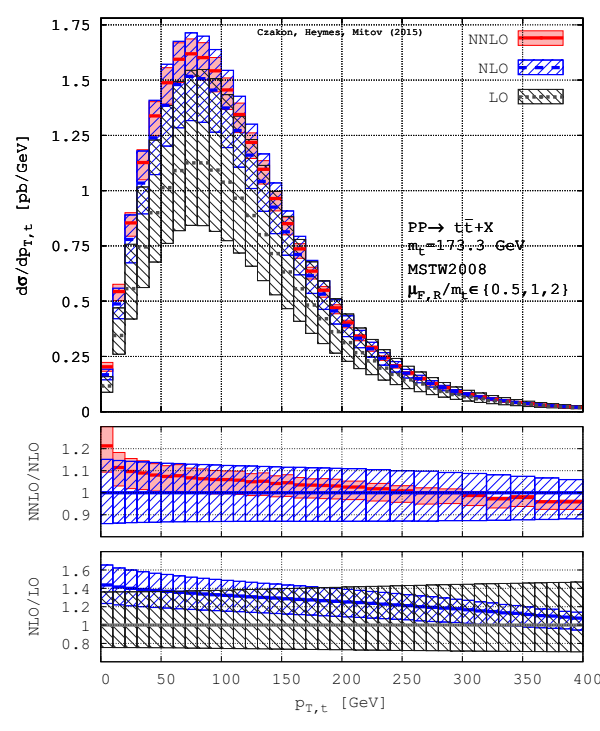

Figure 1: The top quark transverse momentum at LO, NLO and NNLO, and the associated K-factors (LHC, $\sqrt{S}=8 \mathrm{TeV}$ ). Figure from ref. [4]. ATLAS and CMS have shown an unsatisfactory agreement between data and NLO-matched event

\footnotetext{
${ }^{1}$ It has to be noticed though, that a fixed renormalization and factorization scale is used in these results.
} 
generators, with the latter overshooting the measured cross section. By including NNLO corrections, the agreement is significantly improved, as also shown in ref. [4]. An important refinement of these differential results will be its combination with the NNLO differential computation of the top decay, that was performed in refs. [5, 6].

For completeness I also recall that significant progress towards the computation of NNLO QCD corrections to total and differential $t \bar{t}$ cross sections was also made by other groups [7, 8]. For instance the cross section $d \sigma / d p_{T, t \bar{t}}$ for the transverse momentum of the top pair is now known in the small $p_{T}$ limit at the next-to-next-to-leading logarithmic order $[9,10]$ : steps towards a generalization of the " $q_{T}$-subtraction" method to perform a NNLO computation with colored final states have started to be taken [8], thanks to the availability of these results.

\subsection{All-order results}

When the $t \bar{t}$ system is produced just above threshold, effects due to multiple soft-gluon radiation become relevant. In this phase-space region large logarithmically-enhanced corrections of the form $\alpha_{s}^{n} \log ^{m} X_{s}$ appear, where $X_{s}$ is a kinematic function that vanishes when the $t \bar{t}$ system is produced exactly at threshold. Thanks to factorization properties of phase space and production matrix elements in the soft region (the latter being not trivial for $t \bar{t}$ due to color flow), the perturbative expansion can be reorganized suitably, by computing systematically the log-enhanced terms, and resumming them to all orders.

Several groups performed soft-gluon threshold resummation at next-to-next-to-leading logarithmic (NNLL) accuracy [11, 12, 13, 14, 15], using different formalisms and different functional forms for $X_{S}$, and in one case also resumming Coulomb effects [14]. NNLL resummation has also been matched to the exact NNLO computation mentioned in sec. 2.1: this accuracy represent the state of the art for $\sigma_{t o t}$, and is available in public codes as TOP ++ [1] and TOP iXS [16].

Resummed results can also be used to guess the size of the first unknown term at fixed order: until the exact NNLO result was available, approximate NNLO results have been computed by several groups. Nowadays the natural development is to try and compute $\sigma_{t o t}$ at approximate $\mathrm{N}^{3} \mathrm{LO}$. This has been achieved, using different approaches, in ref. [17], as well as in ref. [18] (in the latter paper, high-energy resummation was used together with threshold resummation).

\subsection{Fully differential computations, including top-quark decays}

Since top quarks can not be detected, $\sigma_{t o t}$ and differential distributions for stable top quarks can not be directly compared with data without some extrapolation. In this respect, differential results that include also the final state decay products are of fundamental importance, and are also a central ingredient for the latest developments of modern event generators, to be discussed in sec. 3. The more accurate fixed-order results for describing the fully exclusive final state arising from "top pair" production and decay are those presented, a while ago, in refs. [19, 20, 21, 22, 23]. Despite there being differences among the computations, all these papers essentially contain fully differential NLO results for the process ${ }^{2} p p \rightarrow W^{+} W^{-} b \bar{b}$, where all offshellness effects as well as interference among double-, singly- and non-resonant diagrams are taken into account exactly up

\footnotetext{
${ }^{2}$ Throughout the document with this notation it is implied that leptonic $W$ decays are included.
} 
to NLO in QCD. A remarkable result was recently obtained in ref. [24], where the same effects were also computed at NLO for the process $p p \rightarrow W^{+} W^{-} b \bar{b}+1$ jet.

It is worth stressing that two of the aforementioned computations (namely [22] and [23]) were performed in the "4-flavor" scheme, i.e. the $b$ quarks are considered massive, hence results are finite also in the limit of vanishing $p_{T}$ for one (or both) $b$ quarks (as well as for unresolved $b$-tagged jets). Therefore these computations allow a clean comparison among theory predictions (at NLO) and data for the experimental signature traditionally called "single-top $W t$ " production: since arbitrary cuts can be safely placed on $b$ jets, consistent results can be obtained for the same final state detected experimentally ( $2 b$-tagged jets vs. e.g. $1 b$-tag and $1 b$-veto), thereby avoiding the ambiguities intrinsically present when a separation between top-pair and $W t$ production is attempted theoretically. An experimental analysis dedicated to a comparison among these computations and data would certainly be very valuable.

\section{Recent improvements in event generators}

Fully exclusive Monte Carlo event generators based on parton-shower algorithms are used ubiquitously in experimental searches, hence their importance for LHC phenomenology doesn't need to be stressed in this short review. A substantial step to improve the accuracy of these simulation tools has been made more than a decade ago, when methods to consistently match NLO QCD computations with parton showers algorithms were devised (NLO+PS). Since then, an enormous progress took place in this field: all important processes of the type $p p \rightarrow t \bar{t}+X$ can be simulated at NLO+PS, thanks to the developments of fully- or partially-automated frameworks [25, 26, 27, 28, 29]. Among them, MadGraph5_aMC@NLO [26] deserves to be specially mentioned, being it the only one which is currently fully automated in the strictest sense. For other frameworks, if a specific process of interest is not publicly available, its NLO+PS simulation can be obtained with minor efforts, by linking against external codes (typically, to obtain 1-loop amplitudes with large multiplicity). Nowadays this can be done straightforwardly, using standard interfaces developed specifically for this purpose [30, 31].

There are currently two very active research topics in the community of Monte Carlo developers that are relevant for top pair production at hadron colliders: the consistent inclusion of offshellness effects in presence of intermediate resonances decaying into colored particles, and the merging of NLO+PS simulations for different jet multiplicities. I will review them in turn, with particular emphasis on the former.

\subsection{Simulation of $p p \rightarrow W^{+} W^{-} b \bar{b}$ at NLO+PS accuracy}

Before turning to the explanation of the theoretical issues presently addressed by the community, I want to recall that a major application where a NLO+PS simulation of of $p p \rightarrow W^{+} W^{-} b \bar{b}$ can have an impact is in the determination of the top mass, at least for the techniques where the kinematics of visible particles from top-decay is used for this purpose (see [32] for a recent review).

The problem with the simulation of $W^{+} W^{-} b \bar{b}$ production can be stated as follows: unless special care is taken, when NLO correction to the decay are included in NLO+PS tools, the intermediate top-quark virtuality is not preserved. If this happens, non-physical distortion can potentially 
show up in kinematic distributions. Although there are issues also with the MC@NLO method (see for instance [33]), from this point onward I'll focus on the POWHEG approach.

Before entering into details, a remark is due: it is legitimate to ask whether the issues discussed below are really relevant for practical purposes, especially because NLO+PS results for the $W^{+} W^{-} b \bar{b}$ final state (with offshellness and interference effects) were obtained with PowHel in ref. [34], and no particular problems were noticed by the authors. The definitive answer can only be given by developing more refined tools and performing careful comparisons among them and against older approaches.

The POWHEG "master formula" to generate a resolved emission reads

$$
d \sigma=d \Phi_{B} d \Phi_{\mathrm{rad}} \bar{B}\left(\Phi_{B}\right) \frac{R\left(\Phi_{B}, \Phi_{\mathrm{rad}}\right)}{B\left(\Phi_{B}\right)} \exp \left[-\int \frac{R\left(\Phi_{B}, \Phi_{\mathrm{rad}}\right)}{B\left(\Phi_{B}\right)} d \Phi_{\mathrm{rad}}\right] .
$$

The concept of "underlying Born" phase space $\left(\Phi_{B}\right)$ is central in POWHEG, and we assume the reader to be familiar with it: once a point in $\Phi_{B}$ is picked, according to the weight $\bar{B}$, the hardest emission (a point in $\left(\Phi_{B}, \Phi_{\text {rad }}\right)$ ) is generated according to the POWHEG Sudakov. The mapping $\Phi_{B} \rightarrow\left(\Phi_{B}, \Phi_{\text {rad }}\right)$ is the same as the one used to perform the subtraction of singularities present in the $R$ term contained within the $\bar{B}$ function, and it depends on the singular region at hand [35]. Two problems are present: ${ }^{3}$

1. In the standard POWHEG BOX algorithm, the phase space region associated to final-state gluon emission off the $b$-quark would be handled by a mapping that, in general, does not preserve the virtuality of the intermediate resonance, i.e. $m_{W b}^{2}\left(\Phi_{B}\right) \neq m_{W b g}^{2}\left(\Phi_{B}, \Phi_{\mathrm{rad}}\right)$. The problem is manifest: unless $m_{b g}^{2} \ll \Gamma_{t} E_{b g}, R$ and $B$ will not be on the resonance peak at the same time, hence the ratio $R / B$ can become large when $R$ is on peak and $B$ is not, yielding a "Sudakov suppression" that is spurious, since the $\left(\Phi_{B}, \Phi_{\text {rad }}\right)$ kinematics would be far from the true QCD singularity. Quantitatively, one expects the mass profile of the $b$-jet to be distorted when $m_{\text {jet }}^{2} \sim E_{b} \Gamma_{t}$.

2. A further problem can arise during the parton-showering stage: from the second emission onward, the shower should be instructed to preserve the mass of the resonances. This could be done easily if there was an unique mechanism to "assign" the radiation to a given resonance. For processes where interference is present, no obvious choice is possible.

An intermediate solution to the previous issues was presented in ref. [36], where a fully consistent NLO+PS simulation for $W^{+} W^{-} b \bar{b}$ production in the narrow-width limit was obtained. Offshellenss and interference effects were implemented in an approximate way, as follows:

a. By using matrix elements in the narrow-width limit, real and virtual corrections for production and decay can be clearly separated, i.e. no interference arises. This also allows for a non-ambiguous "resonance assignment" for final-state radiation.

b. For radiation in the decay, $\Phi_{\text {rad }}$ is generated by first boosting momenta in the resonance rest-frame. In this way, the intermediate virtuality is the same for $\Phi_{\mathrm{rad}}$ and $\left(\Phi_{B}, \Phi_{\mathrm{rad}}\right)$.

\footnotetext{
${ }^{3}$ Extended explanations can be found in refs. [36, 37].
} 
c. The phase space integration, and the event generation, spans also over the off-shell regions. A projection onto an on-shell kinematics, tested extensively, allows to use the NLO on-shell amplitudes (computed in refs. [38, 39]).

d. From the off-shell phase space, a reweighting of the $\bar{B}$ function is performed using the LO exact results (where finite width and non-double-resonant diagrams are fully included).

The above expedients allow the construction of a NLO+PS generator where the theoretical problems mentioned above are solved, and offshellness effects are included approximately. A further issue was addressed: by default in POWHEG only the hardest emission is generated. However, for the $t \bar{t}$ process, emissions from decay are rarely the hardest, hence they would be dealt with by the shower most of the time, despite the previous improvements. In ref. [36] a procedure to keep, at the same time, the initial state radiation as well as those from decaying resonances was implemented, to alleviate the aforementioned issue. An example of the results is illustrated in fig. 2, where the impact of the new POWHEG BOX generator is shown on an "endpoint" observable typically used to extract $m_{t}$.

Finally, I want to mention that, shortly after the

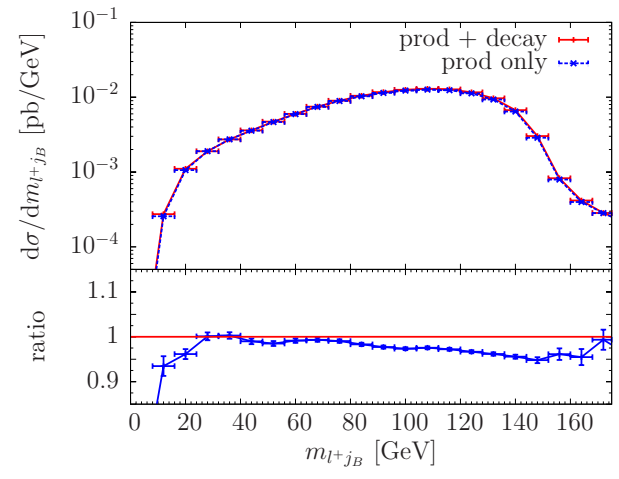

Figure 2: Invariant mass distribution of the charged lepton and $b$-flavored jet at $\mathrm{NLO}+\mathrm{PS}$ including correction for production only or for production and decay (LHC, $\sqrt{S}=8 \mathrm{TeV}$ ), with the method described in ref. [36]. Figure adapted from ref. [36]. conference, further substantial progress was presented in ref. [37]. A method to handle exactly the complete matrix elements also at NLO was developed, by partially using some of the improvements in ref. [36] but also generalizing substantially the partition of phase space into singular regions and the associated subtraction scheme. Although results were published only for single-top, the method is fully general, and its application to $W^{+} W^{-} b \bar{b}$ is in progress [40].

\subsection{Multijet merging at NLO}

At large collision energies, a significant fraction of $t \bar{t}$ events is produced in association with one or more jets. At times a tool describing several jet multiplicities in a single event sample is needed. A typical example is when " $H_{T}$ " variables are used, as is often the case in BSM searches.

The CKKW-L and MLM-merging methods succesfully address this issue at LO. Since this accuracy will become a limiting factor for precision studies, it is desirable to extend these methods to NLO ("NLOPS multijet merging"). Reaching such accuracy is a non-trivial theoretical challenge, since it requires a detailed understanding of the interplay among resummation and fixed order effects in NLO+PS simulations. Several approaches were proposed in the literature over the last three years. In the context of top-pair production, so far results were published only using the MEPS@NLO [41] and FxFx [42] merging methods. ${ }^{4}$ The measurement of QCD activity in $t \bar{t}$ events

\footnotetext{
${ }^{4}$ Phenomenological studies performed by the original authors can also be found in refs. [43, 44] and ref. [26]. Moreover a thorough comparison where other approaches are also included will be presented in the proceedings of the 2015 "Physics at TeV Colliders" workshop [45].
} 
will allow to test these tools against data in "SM dominated" regions, thereby providing a robust assessment of the accuracy that can be assumed when they are used for BSM searches.

\section{References}

[1] M. Czakon, P. Fiedler and A. Mitov, Phys. Rev. Lett. 110, 252004 (2013)

[2] M. Czakon, Phys. Lett. B 693, 259 (2010)

[3] M. Czakon, P. Fiedler and A. Mitov, Phys. Rev. Lett. 115, no. 5, 052001 (2015)

[4] M. Czakon, D. Heymes and A. Mitov, arXiv:1511.00549 [hep-ph].

[5] J. Gao, C. S. Li and H. X. Zhu, Phys. Rev. Lett. 110, no. 4, 042001 (2013)

[6] M. Brucherseifer, F. Caola and K. Melnikov, JHEP 1304, 059 (2013)

[7] G. Abelof, A. Gehrmann-De Ridder and I. Majer, JHEP 1512, 074 (2015)

[8] R. Bonciani, S. Catani, M. Grazzini, H. Sargsyan and A. Torre, Eur. Phys. J. C 75, no. 12, 581 (2015)

[9] H. T. Li, C. S. Li, D. Y. Shao, L. L. Yang and H. X. Zhu, Phys. Rev. D 88, 074004 (2013)

[10] S. Catani, M. Grazzini and A. Torre, Nucl. Phys. B 890, 518 (2014)

[11] M. Czakon, A. Mitov and G. F. Sterman, Phys. Rev. D 80, 074017 (2009)

[12] N. Kidonakis, Phys. Rev. D 82, 114030 (2010)

[13] V. Ahrens, A. Ferroglia, M. Neubert, B. D. Pecjak and L. L. Yang, JHEP 1009, 097 (2010)

[14] M. Beneke, P. Falgari, S. Klein and C. Schwinn, Nucl. Phys. B 855, 695 (2012)

[15] M. Cacciari, M. Czakon, M. Mangano, A. Mitov and P. Nason, Phys. Lett. B 710, 612 (2012)

[16] M. Beneke, P. Falgari, S. Klein, J. Piclum, C. Schwinn, M. Ubiali and F. Yan, JHEP 1207, 194 (2012)

[17] N. Kidonakis, Phys. Rev. D 90, no. 1, 014006 (2014)

[18] C. Muselli, M. Bonvini, S. Forte, S. Marzani and G. Ridolfi, JHEP 1508, 076 (2015)

[19] A. Denner, S. Dittmaier, S. Kallweit and S. Pozzorini, JHEP 1210, 110 (2012)

[20] G. Bevilacqua, M. Czakon, A. van Hameren, C. G. Papadopoulos and M. Worek, JHEP 1102, 083 (2011)

[21] G. Heinrich, A. Maier, R. Nisius, J. Schlenk and J. Winter, JHEP 1406, 158 (2014)

[22] R. Frederix, Phys. Rev. Lett. 112, no. 8, 082002 (2014)

[23] F. Cascioli, S. Kallweit, P. Maierhöfer and S. Pozzorini, Eur. Phys. J. C 74, no. 3, 2783 (2014)

[24] G. Bevilacqua, H. B. Hartanto, M. Kraus and M. Worek, arXiv:1509.09242 [hep-ph].

[25] S. Alioli, P. Nason, C. Oleari and E. Re, JHEP 1006, 043 (2010)

[26] J. Alwall et al., JHEP 1407, 079 (2014)

[27] S. Hoeche, F. Krauss, M. Schonherr and F. Siegert, JHEP 1209, 049 (2012)

[28] S. Platzer and S. Gieseke, Eur. Phys. J. C 72, 2187 (2012)

[29] A. Kardos, C. Papadopoulos and Z. Trocsanyi, Phys. Lett. B 705, 76 (2011) 
[30] T. Binoth et al., Comput. Phys. Commun. 181, 1612 (2010)

[31] S. Alioli et al., Comput. Phys. Commun. 185, 560 (2014)

[32] G. Corcella, arXiv:1511.08429 [hep-ph].

[33] A. Papanastasiou, these proceedings.

[34] M. V. Garzelli, A. Kardos and Z. Trocsanyi, JHEP 1408, 069 (2014)

[35] S. Frixione, P. Nason and C. Oleari, JHEP 0711, 070 (2007)

[36] J. M. Campbell, R. K. Ellis, P. Nason and E. Re, JHEP 1504, 114 (2015)

[37] T. Ježo and P. Nason, JHEP 1512, 065 (2015)

[38] J. M. Campbell and R. K. Ellis, J. Phys. G 42, no. 1, 015005 (2015)

[39] S. Badger, R. Sattler and V. Yundin, Phys. Rev. D 83, 074020 (2011)

[40] T. Ježo, J. Lindert, P. Nason, C. Oleari and S. Pozzorini, in progress.

[41] S. Hoeche, F. Krauss, M. Schonherr and F. Siegert, JHEP 1304, 027 (2013)

[42] R. Frederix and S. Frixione, JHEP 1212, 061 (2012)

[43] S. Hoeche, J. Huang, G. Luisoni, M. Schoenherr and J. Winter, Phys. Rev. D 88, 014040 (2013)

[44] S. Hoeche, F. Krauss, P. Maierhoefer, S. Pozzorini, M. Schonherr and F. Siegert, Phys. Lett. B 748, 74 (2015)

[45] https://phystev.chrs.fr/ 